\title{
Expansile duraplasty and obex exploration compared with bone-only decompression for Chiari malformation type $I$ in children: retrospective review of outcomes and complications
}

\author{
${ }^{*}$ Chibawanye I. Ene, MD, PhD, ${ }^{1}$ Anthony C. Wang, MD, ${ }^{2}$ Kelly L. Collins, MD, ${ }^{3}$ \\ Robert H. Bonow, MD, ${ }^{1,4}$ Lynn B. McGrath, MD, ${ }^{1}$ Sharon J. Durfy, PhD, ${ }^{1}$ Jason K. Barber, MS, ${ }^{1}$ \\ and Richard G. Ellenbogen, MD1
}

1Department of Neurological Surgery and ${ }^{4}$ Harborview Injury Prevention Research Center, University of Washington, Seattle, Washington; 'Department of Neurosurgery, University of California, Los Angeles, California; and 'UPMC Children's Hospital of Pittsburgh, Pennsylvania

OBJECTIVE While a select population of pediatric patients with Chiari malformation type I (CM-I) remain asymptomatic, some patients present with tussive headaches, neurological deficits, progressive scoliosis, and other debilitating symptoms that necessitate surgical intervention. Surgery entails a variety of strategies to restore normal CSF flow, including increasing the posterior fossa volume via bone decompression only, or bone decompression with duraplasty, with or without obex exploration. The indications for duraplasty and obex exploration following bone decompression remain controversial. The objective of this study was to describe an institutional series of pediatric patients undergoing surgery for $\mathrm{CM}-\mathrm{I}$, performed by a single neurosurgeon. For patients presenting with a syrinx, the authors compared outcomes following bone-only decompression with duraplasty only and with duraplasty including obex exploration. Clinical outcomes evaluated included resolution of syrinx, scoliosis, presenting symptoms, and surgical complications.

METHODS A retrospective review was conducted of the medical records of 276 consecutive pediatric patients with CM-I operated on at a single institution between 2001 and 2015 by the senior author. Imaging findings of tonsillar descent, associated syrinx (syringomyelia or syringobulbia), basilar invagination, and clinical assessment of CM-I-attributable symptoms and scoliosis were recorded. In patients presenting with a syrinx, clinical outcomes, including syrinx resolution, symptom resolution, and impact on scoliosis progression, were compared for three surgical groups: boneonly/posterior fossa decompression (PFD), PFD with duraplasty (PFDwD), and PFD with duraplasty and obex exploration (PFDwDO).

RESULTS PFD was performed in $25 \%$ of patients (69/276), PFDwD in $18 \%$ of patients (50/276), and PFDwDO in $57 \%$ of patients (157/276). The mean follow-up was $35 \pm 35$ months. Nearly half of the patients $(132 / 276,48 \%)$ had a syrinx. In patients presenting with a syrinx, PFDwDO was associated with a significantly higher likelihood of syrinx resolution relative to PFD only (HR 2.65, $p=0.028$ ) and a significant difference in time to symptom resolution (HR 2.68, $p=0.033$ ). Scoliosis outcomes did not differ among treatment groups $(p=0.275)$. Complications were not significantly higher when any duraplasty (PFDwD or PFDwDO) was performed following bone decompression ( $p>0.99)$.

CONCLUSIONS In this series of pediatric patients with CM-I, patients presenting with a syrinx who underwent expansile duraplasty with obex exploration had a significantly greater likelihood of syrinx and symptom resolution, without increased risk of CSF-related complications, compared to those who underwent bone-only decompression.

https://thejns.org/doi/abs/10.3171/2020.6.PEDS20376

KEYWORDS Chiari I malformation; syringomyelia; duraplasty; obex; scoliosis

ABBREVIATIONS BAEP = brainstem auditory evoked potential; $\mathrm{CM}-\mathrm{I}=$ Chiari malformation type I; $\mathrm{MEP}=$ motor evoked potential; $\mathrm{PFD}=$ posterior fossa decompression; PFDwD = PFD with duraplasty; PFDwDO = PFD with duraplasty and obex exploration; PRSRC $=$ Park-Reeves Syringomyelia Research Consortium; SSEP = somatosensory evoked potential.

SUBMITTED May 7, 2020. ACCEPTED June 29, 2020.

INCLUDE WHEN CITING Published online October 30, 2020; DOI: 10.3171/2020.6.PEDS20376.

${ }^{*}$ C.I.E. and A.C.W. contributed equally to this work. 
$\mathrm{C}$ HIARI malformation type I (CM-I) is an anatomicalphysiological anomaly of uncertain etiology that can cause cerebellar dysfunction, brainstem compression, cranial nerve dysfunction, spinal cord injury, and CSF flow disruption. ${ }^{1}$ Resultant pressure-type headaches exacerbated by straining, eye movement abnormalities, central sleep apnea, swallowing dysfunction, imbalance, dyscoordination, and sensory dysfunction are common symptoms at presentation. ${ }^{2-5}$ Among children undergoing MRI, approximately $0.8 \%-3.7 \%$ have cerebellar tonsil descent $\geq 5 \mathrm{~mm},{ }^{6-8}$ the most commonly used radiographic screening criterion for CM-I. In cases of asymptomatic presentation, the incidental discovery of pathologic hydromyelia/syringomyelia (syrinx) is common. Treatment for this condition is aimed at symptom management, neural decompression, and restoration of normal CSF flow dynamics.

Symptomatic presentation of CM-I, frequently with headache, is most common in the 3rd decade of life, whereas asymptomatic presentation associated with a syrinx appears more commonly in children. ${ }^{9}$ Symptomatic presentation in childhood or adulthood indicates, but does not necessitate, surgical intervention; surgical treatment is indicated when presentation includes a syrinx with or without symptoms. In this study, the authors describe an institutional series of consecutive pediatric patients undergoing surgery for CM-I performed by a single neurosurgeon. For patients presenting with a syrinx, we compare clinical outcomes following bone-only decompression, decompression with autologous duraplasty, and decompression with duraplasty and obex exploration. Clinical outcomes evaluated in patients presenting with a syrinx include resolution of syrinx, progression of scoliosis, and symptomatic improvement.

\section{Methods}

After IRB approval, medical records were reviewed for all patients under the age of 20 years who underwent surgery for the treatment of CM-I by a single surgeon (R.G.E.) from May 2001 through December 2015. Patients undergoing reoperations were excluded from the analysis. Patients were diagnosed with CM-I on the basis of MRI findings of tonsillar descent and morphology, associated syrinx, basilar invagination, and clinical assessment of CM-I-attributable symptoms such as tussive headaches, central sleep apnea, and scoliosis.

Tonsillar descent was assessed by identifying the line from the basion to opisthion, then measuring from this line to the inferior margin of the cerebellar tonsils on sagittal MRI cuts. For this study, a syrinx was defined as a T1hypointense, T2-hyperintense intrinsic spinal cord cystic lesion $\geq 3 \mathrm{~mm}$ in diameter on MRI. Clinical evaluations were performed by at least one pediatric neurosurgeon and one advanced practice provider (nurse practitioner). Patients were considered symptomatic if the treating neurosurgeon considered any of the patient's symptoms attributable to CM-I or syrinx.

Patients were categorized into three surgical groups: 1) bone-only posterior fossa decompression (PFD), 2) PFD with expansile duraplasty (PFDwD), and 3) PFD with duraplasty and obex exploration for lysis of tissue veil ob- structing the obex (PFDwDO). The decision to perform PFD versus PFDwD or PFDwDO was based on surgeon preference. The surgical technique involved suboccipital craniotomy and $\mathrm{C} 1$ laminectomy for all patients. No patients underwent laminectomy below $\mathrm{C} 1$. The suboccipital craniectomy was typically $3.5-4.0 \mathrm{~cm}$ in width and $1.5-$ $2.0 \mathrm{~cm}$ in height, with the lateral aspect of the craniectomy extending to the lateral margins of the foramen magnum. ${ }^{10}$ In PFD cases, the posterior atlantooccipital ligament was incised in the midline and dissected off of the dura, and the external layer of dura was thinned until no constriction of dura at the level of the foramen magnum was visible. In cases undergoing bone-only decompression, ultrasonography was used to assess restoration of normal pulsatility of the cerebellar tonsils and CSF flow through the foramen magnum once decompression was complete.

For patients who underwent intradural exploration, we utilized a linear dural incision extending from the superior aspect of the $\mathrm{C} 2$ lamina to $1 \mathrm{~cm}$ or more above the craniovertebral junction up to the level of the cerebellar hemisphere. The cerebellar tonsils were then elevated using gentle pial cauterization, until the choroid plexus of the fourth ventricle was visible. Successful obex exploration was defined by either finding a patent obex, or lysis of any arachnoid or scar tissue overlying the obex such that the obex could be confirmed to be patent by both visualization and palpation. Any veil obstructing the obex was sharply lysed with an arachnoid knife such that the obex was confirmed to be patent by palpation with a blunttipped microsurgical nerve hook and direct visualization. A pericranial autograft was then used to perform an oblong expansile patch duraplasty to augment the durotomy and reconstitute craniocaudal CSF flow.

For all patients, age at surgery, sex, tonsillar descent, syrinx characteristics, scoliosis characteristics, and the presence of basilar invagination were recorded. Aspects of surgery recorded included expansile autologous pericranial duraplasty; obex exploration with tissue veil lysis; use of intraoperative sonography; intraoperative electrophysiological monitoring data, including motor evoked potentials (MEPs), somatosensory evoked potentials (SSEPs) and brainstem auditory evoked potentials (BAEPs); and complications including postoperative CSF leak, wound infection, or neurological deficits. Postoperative symptom resolution at immediate and latest follow-up, progression of syringomyelia, and radiographic progression of scoliosis (worsening Cobb measurement per the radiologist and orthopedic surgery follow-up) were obtained from the medical record. Syrinx resolution was based on independent radiology interpretation and defined as the first follow-up MRI scan demonstrating radiological absence of a previously described syrinx. Symptom resolution was defined based on patient-reported absence of symptoms during routine follow-up.

\section{Statistical Analysis}

In patients presenting with a syrinx, pairwise differences in rates of syrinx and symptom resolution within the three treatment groups was evaluated for statistical significance using proportion-odds regression modeling, adjusting for age and sex as potential confounders. Scolio- 
TABLE 1. Patient demographics and clinical variables for surgical groups

\begin{tabular}{|c|c|c|c|c|c|}
\hline \multirow[b]{2}{*}{ Variable } & \multirow[b]{2}{*}{ Total } & \multicolumn{4}{|c|}{ Surgery Type } \\
\hline & & PFD & PFDwD & PFDwDO & $p$ Value \\
\hline No. of patients & 276 & $69(25)$ & $50(18)$ & $157(57)$ & \\
\hline Mean age (SD), yrs & $10.0(5.0)$ & $8.0(5.0)$ & $10.5(4.9)$ & $10.7(4.8)$ & $<0.001$ \\
\hline \multicolumn{6}{|l|}{ Sex } \\
\hline Male & $123(45)$ & $38(55)$ & $20(40)$ & $65(41)$ & 0.126 \\
\hline Female & $153(55)$ & $31(45)$ & $30(60)$ & $92(59)$ & \\
\hline Mean tonsil ectopia (SD), mm & $12.1(6.9)$ & $8.7(3.8)$ & $12.0(6.8)$ & $13.6(7.4)$ & $<0.001$ \\
\hline \multicolumn{6}{|l|}{ Syrinx } \\
\hline No & $144(52)$ & $52(75)$ & $29(58)$ & $63(40)$ & $<0.001$ \\
\hline Yes & $132(48)$ & $17(25)$ & $21(42)$ & $94(60)$ & \\
\hline Cervical level & $105(80)$ & $10(59)$ & $15(71)$ & $80(85)$ & \\
\hline Thoracic level & $111(84)$ & $16(94)$ & $15(71)$ & $80(85)$ & \\
\hline Lumbar level & $36(27)$ & $5(29)$ & $3(14)$ & $28(30)$ & \\
\hline \multicolumn{6}{|l|}{ Scoliosis } \\
\hline No & $233(84)$ & $65(94)$ & $42(84)$ & $126(80)$ & 0.021 \\
\hline Yes & $43(16)$ & $4(6)$ & $8(16)$ & $31(20)$ & \\
\hline Mean follow-up (SD), mos & $35(35)$ & $41(40)$ & $38(40)$ & $32(31)$ & 0.598 \\
\hline
\end{tabular}

sis outcome was assessed used a Mann-Whitney exact test with no evidence of potential confounding effects. Further adjustment for a 5\% family-wise error rate was performed per the Benjamini-Hochberg procedure ${ }^{11}$ across all 7 pairwise comparisons. Differences in rates of complications were evaluated using Fisher's exact test with no further adjustment. All analyses were performed using SPSS (version 19, IBM Corp.) and SAS (version 9.3, SAS Institute Inc.). The impact of surgeon selection bias on the primary aims was assessed using a two-group propensity-weighted approach, with the goal of effectively balancing out observed differences in key covariates among the surgery groups. Unlike case-control matching in which unmatched subjects are excluded, in the propensity-weighted approach all subjects are utilized and given statistical weight in proportion to how closely they resemble members in the opposite group to which they were actually assigned. Applying this to our primary aim, a separate propensity model for each two-group comparison was constructed using age, sex, and tonsil width to model the retrospective probability of each subject being assigned to their actual treatment group. The modeling was conducted using the boosted regression algorithm contained within the TWANG Shiny App available at the RAND Corporation website. ${ }^{12}$ The weights used in the analysis were calculated by taking the predicted probabilities from the boosted regression algorithm and inverting (reciprocating) them such that a low probability corresponded to a large weight. These weights were then standardized so that the sum of all propensity weights within each treatment group remained equal to the original number of subjects, thereby not artificially altering the sample size due to the weighting. All primary analyses were then performed in exactly the same way but with the propensity weights applied.

\section{Results}

\section{Study Demographics}

A total of 276 patients met inclusion criteria, had at least one postoperative follow-up visit (mean 35 months) with imaging, and were included in the analysis. Demographic and clinical characteristics are presented in Table 1. A total of 5 patients $(2 \%)$ had basilar invagination.

As shown in Table 1, PFD was performed in 69 patients (25\%). PFDwD was performed in 50 patients $(18 \%)$. PFDwDO was performed in 157 patients (57\%). Figure 1 shows intraoperative views of 2 surgical cases, depicting the obex prior to tissue veil lysis and following successful tissue veil lysis. Intraoperatively, MEPs and SSEPs were performed in 270 cases (98\%). MEP changes were noted in 3 cases (1\%), while SSEP changes occurred in 7 cases (2.5\%). BAEPs were performed in 102 cases (37\%), with changes occurring in 2 of these (2\%). All changes were transient and did not result in any postoperative neurological deficits.

A syrinx was identified in 132 (48\%) of 276 patients; of this total, 105 involved the cervical segment, 111 included the thoracic segment, and 36 involved the lumbar segment of the spinal cord, with many affecting multiple segments. Scoliosis was identified in 43 (16\%) of 276 patients (Table 1). Duraplasty with or without obex exploration was more likely to be performed on older children $(\mathrm{p}<0.001)$, patients with larger tonsillar ectopia $(\mathrm{p}<0.001)$, and patients presenting with a syrinx $(\mathrm{p}<0.001$; Table 1$)$.

\section{Outcomes Following Duraplasty and Obex Exploration in Patients Presenting With Syrinx}

Compared with PFD, patients with syrinx undergoing PFDwD did not exhibit a significantly increased likelihood 

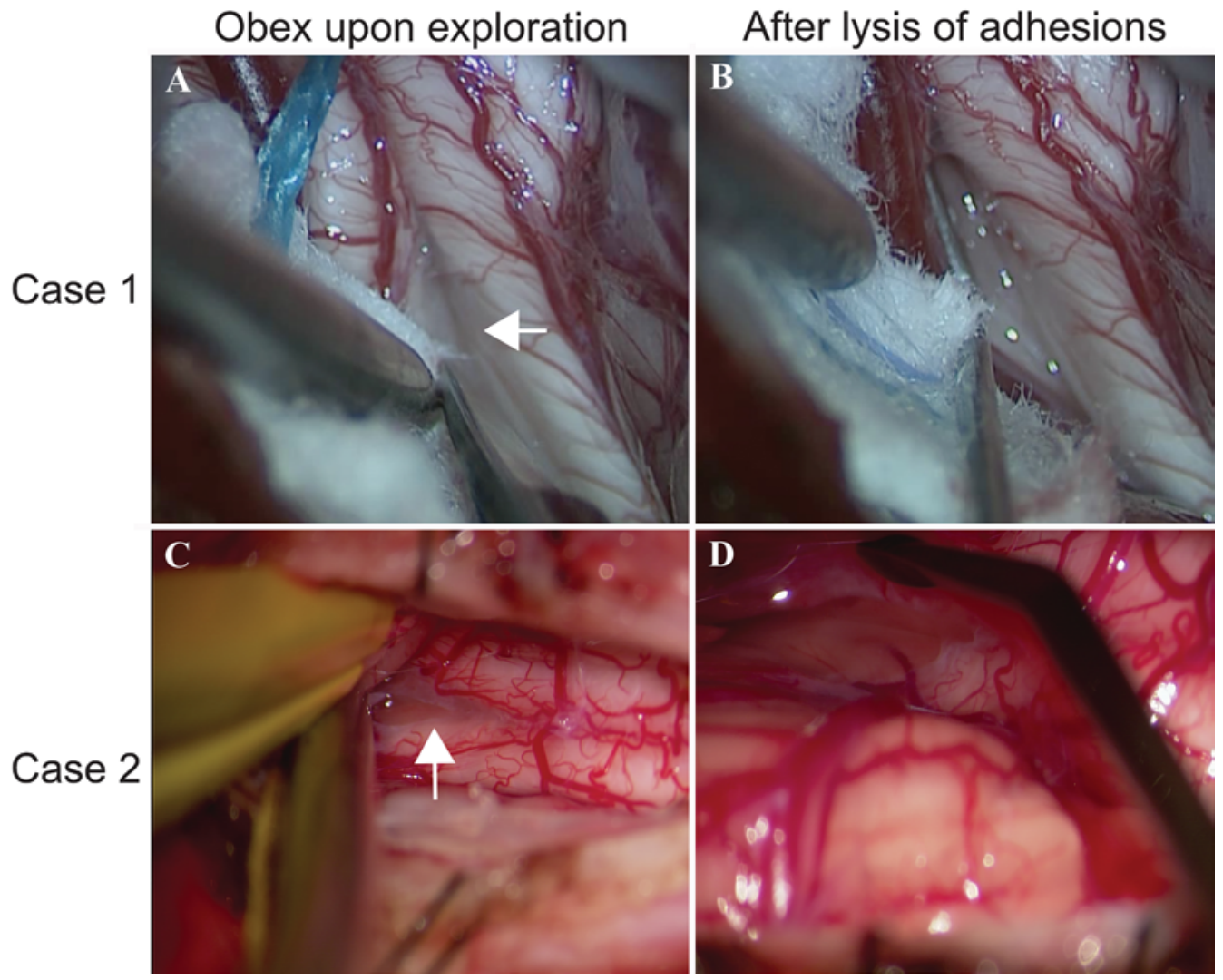

FIG. 1. Obex exploration and lysis of overlying tissue veil. Case 1: Intraoperative view with a microscope demonstrating the obex prior to tissue veil lysis (A) and following tissue veil lysis (B). Case 2: The obex prior to tissue veil lysis (C) and following tissue veil lysis (D). Arrows indicate tissue veil overlying the obex.

of syrinx resolution (HR 1.32, $\mathrm{p}=0.567$; Table 2). Performing a PFDwDO, however, significantly improved the likelihood of syrinx resolution when compared with PFD only (HR 2.65, $\mathrm{p}=0.028$; Table 2). There was no significant difference between PFDwD and PFDwDO in terms of syrinx resolution (HR 1.73, $\mathrm{p}=0.198$; Table 2). There was no significant difference in time to symptom resolution or scoliosis outcomes between treatment groups (Table 2).

\section{Outcomes Following Propensity-Weighted Analysis in Patients Presenting With a Syrinx}

The data in Table 1 indicated a bias to perform PFDwDO in older children, children with larger tonsillar ectopia, and children presenting with a syrinx $(\mathrm{p}<0.001)$. To minimize the influence of these potential confounders on syrinx-related outcomes (resolution of syrinx, symptoms, and scoliosis), we performed additional propensity-weighted analysis to adjust for the impact of age and tonsillar ectopia on the outcomes of patients presenting with a syrinx (Table 2). Performing a PFDwDO in patients presenting with a syrinx significantly improved the likelihood of syrinx resolution when compared to PFD only (HR 2.29, p = 0.033; Table 2). Performing a PFDwDO also shortened the time to symptom resolution in patients presenting with a syrinx (relative to PFD only, HR 2.68, p $=0.033$; Table 2). There was no significant difference in scoliosis outcomes (Table 2).

\section{Complications}

A total of 9 complications (3\%) were observed for the entire cohort of 276 patients (Table 3). There were no significant differences in the number of complications between cases with duraplasty (PFDwD and PFDwDO) or without duraplasty (PFD; $p>0.99$; Table 3). In 69 patients undergoing PFD, there were 2 wound infections (2.9\%), and each was treated with oral antibiotics. In 207 patients undergoing dura opening (PFDwD and PFDwDO), there were 4 wound infections (1.9\%), each treated with reoperation for wound washout; 2 patients $(1 \%)$ with pseudomeningoceles that required lumbar drain placement for treatment; and 1 patient $(0.5 \%)$ who had a minor scalp burn from a monitoring electrode without lasting sequelae. Electrophysiological monitoring did not identify any intraoperative changes that altered positioning or operative management. There were no incidences of postoperative lower cranial neuropathy, or other signs of brainstem dysfunction, such as persistent vomiting or bradycardia, that might be of concern with lysis of an arachnoid veil overlying the obex, observed in any of our patients.

\section{Discussion}

In patients presenting with a syrinx, the indications for duraplasty following bone decompression for CM-I remain controversial. ${ }^{13}$ This topic is currently the subject 
TABLE 2. Outcomes for patients with syrinx

\begin{tabular}{|c|c|c|c|c|c|c|}
\hline \multirow{3}{*}{$\begin{array}{c}\text { Outcomes } \\
\text { (patients w/ syrinx only) }\end{array}$} & & & \multicolumn{4}{|c|}{ Significance } \\
\hline & \multicolumn{2}{|c|}{ Surgery Type } & \multicolumn{2}{|c|}{ Unweighted* } & \multicolumn{2}{|c|}{ Propensity-Weighted $†$} \\
\hline & PFD (n=17) & PFDwD $(n=21)$ & $\mathrm{HR}(95 \% \mathrm{Cl})$ & $p$ Value & $\mathrm{HR}(95 \% \mathrm{Cl})$ & $\mathrm{p}$ Value \\
\hline \multicolumn{3}{|l|}{ Syrinx resolved } & \multirow[t]{4}{*}{$1.32(0.61-2.86)$} & \multirow[t]{4}{*}{0.567} & \multirow[t]{4}{*}{$1.49(0.67-3.28)$} & \multirow[t]{4}{*}{0.471} \\
\hline No & $5(29)$ & $6(29)$ & & & & \\
\hline Yes & $12(71)$ & $15(71)$ & & & & \\
\hline Mean time to resolution (SD), mos & $48(14)$ & $39(13)$ & & & & \\
\hline \multicolumn{3}{|l|}{ Symptoms resolved } & \multirow[t]{4}{*}{$1.02(0.46-2.25)$} & \multirow[t]{4}{*}{0.969} & \multirow[t]{4}{*}{$1.34(0.58-3.09)$} & \multirow[t]{4}{*}{0.577} \\
\hline No & $5(29)$ & $8(38)$ & & & & \\
\hline Yes & $12(71)$ & $13(62)$ & & & & \\
\hline \multirow[t]{2}{*}{ Mean time to resolution (SD), mos } & $46(14)$ & $55(15)$ & & & & \\
\hline & PFD $(n=17)$ & PFDwDO (n = 94) & & & & \\
\hline \multicolumn{3}{|l|}{ Syrinx resolved } & \multirow[t]{4}{*}{$2.65(1.37-5.14)$} & \multirow[t]{4}{*}{0.028} & \multirow[t]{4}{*}{$2.29(1.43-3.64)$} & \multirow[t]{4}{*}{0.033} \\
\hline No & $5(29)$ & $11(12)$ & & & & \\
\hline Yes & $12(71)$ & $83(88)$ & & & & \\
\hline Mean time to resolution (SD), mos & $48(14)$ & $12(3)$ & & & & \\
\hline \multicolumn{3}{|l|}{ Symptoms resolved } & \multirow[t]{4}{*}{$1.66(0.87-3.14)$} & \multirow[t]{4}{*}{0.284} & \multirow{4}{*}{$2.68(1.58-4.53)$} & \multirow[t]{4}{*}{0.033} \\
\hline No & $5(29)$ & $19(20)$ & & & & \\
\hline Yes & $12(71)$ & $75(80)$ & & & & \\
\hline \multirow[t]{2}{*}{ Mean time to resolution (SD), mos } & $46(14)$ & $20(4)$ & & & & \\
\hline & PFDwD $(n=21)$ & PFDwDO (n = 94) & & & & \\
\hline \multicolumn{3}{|l|}{ Syrinx resolved } & $1.73(0.98-3.04)$ & 0.198 & $1.86(1.20-2.89)$ & 0.074 \\
\hline No & $6(29)$ & $11(12)$ & & & & \\
\hline Yes & $15(71)$ & $83(88)$ & & & & \\
\hline Mean time to resolution (SD), mos & $39(13)$ & $12(3)$ & & & & \\
\hline Symptoms resolved & & & $1.34(0.74-2.43)$ & 0.477 & $1.05(0.67-1.64)$ & 0.766 \\
\hline No & $8(38)$ & $19(20)$ & & & & \\
\hline Yes & $13(62)$ & $75(80)$ & & & & \\
\hline Mean time to resolution (SD), mos & $55(15)$ & $20(4)$ & & & & \\
\hline & $\operatorname{PFD}(n=4)$ & PFDwD \& PFDwDO $(n=29)$ & & & & \\
\hline Scoliosis outcomes & & & - & 0.322 & - & 0.275 \\
\hline Improved & $0(0)$ & $6(21)$ & & & & \\
\hline Stable & $1(25)$ & $12(41)$ & & & & \\
\hline Worsened & $3(75)$ & $11(38)$ & & & & \\
\hline Unknown & 0 & 10 & & & & \\
\hline
\end{tabular}

Values are expressed as number (\%) of patients who underwent the specified procedure unless otherwise indicated. Boldface type indicates statistical significance.

* Statistical significance by proportional hazards regression, adjusting for age and sex as potential confounders and further adjusting for multiple comparisons (Benjamini-Hochberg, $n=7)$.

† Statistical significance as above, followed by propensity weighting for age and tonsillar ectopia $(\mathrm{mm})$ across surgical techniques.

of an ongoing randomized multicenter clinical trial from the Park-Reeves Syringomyelia Research Consortium (PRSRC), which compares PFD to PFDwD in pediatric CM-I patients with a syrinx. ${ }^{14}$ Of note, our retrospective analysis precedes the PRSRC and none of the patients in our study are included in the PRSRC.

Prior to joining the consortium, our practice in all CM-I patients presenting with a syrinx evolved from performing bone-only decompression (PFD), to preferentially performing duraplasty (PFDwD), and eventually obex exploration (PFDwDO). This evolution in practice (and selection bias evident in Table 1) is based on the senior author's observation of the impact of scarring overlying the obex/craniovertebral junction on CSF outflow, as previously reported.${ }^{15}$ Here, we analyzed the results of our practice of performing obex exploration in addition to duraplasty for pediatric patients with CM-I presenting with syrinx, with or without scoliosis. We observed that in patients presenting with a syrinx, this practice (compared to PFD) was associated with an increased likelihood of 
TABLE 3. Complications for total patient cohort and each surgical group

\begin{tabular}{|c|c|c|c|c|}
\hline \multirow[b]{2}{*}{ Complication } & \multirow[b]{2}{*}{ Total $(n=276)$} & \multicolumn{3}{|c|}{ Procedure } \\
\hline & & PFD ( $n=69)$ & PFDwD + PFDwDO (n= 207) & p Value \\
\hline Any complication & $9(3.3)$ & $2(2.9)$ & $7(3.4)$ & $>0.99$ \\
\hline Infections & $6(2.2)$ & $2(2.9)$ & $4(1.9)$ & \\
\hline Pseudomeningoceles requiring temporary CSF diversion & $2(0.7)$ & $0(0.0)$ & $2(1.0)$ & \\
\hline Other & $1(0.4)$ & $0(0.0)$ & $1(0.5)$ & \\
\hline
\end{tabular}

CSF diversion = lumbar drain .

Values are expressed as number (\%) of patients who underwent the specified procedure. Statistical significance was determined by Fisher's exact test.

syrinx and symptom resolution without significantly increased risk of complications. Those patients with a syrinx undergoing obex exploration, regardless of whether an obstruction was discovered, had an $88 \%$ chance of syrinx resolution, within an average of 3 months.

The presence of a syrinx attributable to CM-I, with or without symptoms, is often an indication for offering surgical decompression. In a study of 147 patients followed expectantly upon CM-I diagnosis, development of a syrinx occurred in 8 patients, although 5 of these had a "pre-syrinx" or dilated central canal on initial imaging. ${ }^{8}$ In children with CM-I, a Kids' Inpatient Database analysis identified a coincident syrinx in $23.8 \% .{ }^{16}$ Other large series have identified similar incidences of syrinx in association with CM-I, ${ }^{17}$ although the proportion of operated patients with a syrinx is much higher, ${ }^{9}$ consistent with our series in which 141 (48\%) of 294 patients receiving surgery had a syrinx. The relationship between syrinx resolution and symptom improvement also remains controversial. Although radiographic improvement of a syrinx does not correlate temporally with symptom improvement, patients presenting with sensory symptoms or motor weakness had a higher likelihood of having radiographic syrinx improvement postoperatively, ${ }^{18}$ suggesting a relationship between syrinx resolution and symptoms. A recent preliminary report from the multicenter PRSRC prospective study showed that age and PFDwD are independently associated with radiological syrinx improvement in patients presenting with a syrinx..$^{14,19}$ After propensity-weighted analysis to control for age and tonsillar ectopia size as potential confounders of outcomes in the syrinx patient cohort, we found no significant difference in time to syrinx resolution following PFD compared with PFDwD (48 vs 39 months, $\mathrm{p}=0.471$ ). Following PFDwDO, however, we found a significantly improved mean time to syrinx resolution when compared with PFD (12 vs 48 months, $\mathrm{p}=0.033$ ). Furthermore, there is also a shorter time to symptom resolution following PFDwDO relative to PFD (46 vs 20 months, $\mathrm{p}=$ 0.033 ). These results (retrospective and prospective) indicate that performing a duraplasty may be clinically beneficial in the pediatric patient population, but successful obex exploration with lysis of any obstruction of CSF outflow, if safely performed, may further improve rates of syrinx resolution, as well as the amount of time required for resolution without increased risk of complications.

Although the pathophysiology of syrinx formation in CM-I patients remains a debated subject, many theories regarding syrinx formation have driven the surgical man- agement of patients over the last 7 decades. ${ }^{15,20-23}$ Ball and Dayan proposed that the cavitation of a syrinx may be produced by CSF tracking under pressure into the spinal cord from the subarachnoid space along perivascular (VirchowRobin) spaces. ${ }^{20}$ Williams proposed that abnormalities in venous pressure in CM-I patients result in distension of the central canal. ${ }^{23}$ In contrast, Gardner and Angel proposed that persistent "water hammer" arterial pulsation in the setting of an obstructed fourth ventricle results in syrinx development. ${ }^{21}$ Oldfield et al. ${ }^{22}$ and others have demonstrated that progression of a syrinx is associated with occlusion of the subarachnoid space at the foramen magnum..$^{10,15}$ Using phase contrast ${ }^{16}$ and cardiac-gated phase contrast cine MRI, ${ }^{15}$ impaction of the hindbrain in an abnormal posterior fossa has been shown. This compression obstructs the normal flow of CSF in and out of the craniocervical subarachnoid space throughout the cardiac cycle. Based on these clinical and radiographic correlates, many surgeons propose that decompression or enlargement of the posterior fossa to establish normal CSF flow, similar to a normal subject, should be the primary goal of surgery. We hypothesized that selective vulnerability of the spinal cord to certain factors may contribute to the differential development and propagation of syringomyelia, specifically pulsatile CSF pressure, chronic tonsillar impaction and scarring, and disturbances of normal patterns and pathways of CSF flow. ${ }^{15}$ The results of this retrospective analysis suggest that reestablishment of normal CSF flow by addressing these factors, specifically obex exploration with lysis of any obstructive arachnoid scar when present from chronic impaction, results in a higher likelihood of syrinx resolution in pediatric patients with CM-I.

Concurrent scoliosis has been identified in 11.5\%$22.4 \%$ of patients with cerebellar tonsil descent $\geq 5$ mm. ${ }^{16,24}$ This is consistent with our cohort, in which $15 \%$ of patients presented with scoliosis. A matched study comparing syrinx characteristics and scoliotic parameters in the CM-I population with the idiopathic syrinx population showed no significant differences in syrinx/ cord ratio or the sagittal features of scoliosis or neurological deficits associated with the scoliosis. ${ }^{25}$ The impact of decompression on scoliosis progression or improvement remains controversial, with some studies demonstrating no effect and others showing slowed progression. ${ }^{24-27}$ In our cohort, we found no impact of PFDwD or PFDwDO on scoliosis progression relative to PFD even after controlling for age and tonsillar ectopia size $(p=0.275$; Table 2). One study has shown that spinal deformities are 
more likely to improve after PFD in younger patients $\left(<10\right.$ years) with smaller Cobb measurements $\left(<30^{\circ}\right) .^{19}$ Therefore, performing PFD prior to addressing the scoliosis surgically (as in our cohort) may result in objective clinical improvement that minimizes the degree of deformity of the resultant scoliosis. The ongoing multicenter PRSRC prospective study may shed additional light on this relationship. ${ }^{14}$

A meta-analysis of multiple studies in the pediatric population, 5 retrospective and 2 prospective $(n=582$ total patients), showed that PFDwD is associated with a lower risk of reoperation compared with PFD alone and noted a higher likelihood of CSF-related complications with PFDwD (18.5\% vs $1.8 \%$ ) and no significant difference in clinical improvement or decrease in syringomyelia. ${ }^{3}$ We examined complications associated with surgical approaches with and without duraplasty and found no significant differences in CSF-related complications with and without duraplasty. We observed 2 pseudomeningoceles in our duraplasty cohort (1\%), and both resolved with CSF diversion by lumbar drain. Consistent with a similar report utilizing autologous grafts for duraplasty, ${ }^{28}$ we found that duraplasty did not increase the likelihood of infections relative to PFD. Importantly, we did not observe any incidence of neurological complication in any of our cases. Therefore, we no longer routinely use electrophysiological monitoring on routine CM-I decompression cases, unless a patient presents with a neurological deficit associated with the CM-I.

The limitations of this study include its retrospective methodology, and bias for performing a duraplasty with obex exploration in older children, children with larger tonsillar ectopia, and children presenting with a syrinx. This apparent bias is driven by evolution in practice due to the senior author's hypothesis that arachnoid scarring within the obex contributes to CSF outflow obstruction and syrinx formation. To minimize the impact of bias in surgical technique within the patient cohort presenting with a syrinx, we performed a propensity-weighted analysis to control for age and extent of tonsillar ectopia. Prospective analyses that evaluate matched cohorts, however, are superior to a propensity-weighted retrospective study and will be needed to evaluate the comparative efficacy of these surgical techniques in addressing syrinx, scoliosis, and symptom resolution, as well as surgical complications. Generalizability is also questionable, as these are the patients of a single surgeon at a single institution, with limited numbers of patients.

\section{Conclusions}

For pediatric patients with CM-I presenting with a syrinx, expansile autologous pericranial duraplasty with obex exploration significantly increased the likelihood of syrinx and symptom resolution without significantly increasing the risk of CSF-related complications, when compared with patients who received bone-only decompression. A prospective study of large matched cohorts to include duraplasty with lysis of obex obstructions is needed to clearly ascertain the impact of obex exploration on a syrinx, symptoms, and scoliosis in pediatric CM-I patients.

\section{Acknowledgments}

Funding was provided by grant no. R25NS079200 from the National Institute of Neurological Disorders and Stroke, NIH, to Dr. Ene.

\section{References}

1. McClugage SG, Oakes WJ. The Chiari I malformation. $J$ Neurosurg Pediatr. 2019;24(3):217-226.

2. Abel F, Tahir MZ. Role of sleep study in children with Chiari malformation and sleep disordered breathing. Childs Nerv Syst. 2019;35(10):1763-1768.

3. Chatrath A, Marino A, Taylor D, et al. Chiari I malformation in children - the natural history. Childs Nerv Syst. 2019; 35(10):1793-1799.

4. Fuller JC, Sinha S, Caruso PA, et al. Chiari malformations: an important cause of pediatric aspiration. Int J Pediatr Otorhinolaryngol. 2016;88:124-128.

5. Kim SH, Kim HJ, Kim JS. Perverted downward corrective saccades during horizontal head impulses in Chiari malformation. Cerebellum. 2019;18(3):333-339.

6. Aitken LA, Lindan CE, Sidney S, et al. Chiari type I malformation in a pediatric population. Pediatr Neurol. 2009;40(6): 449-454.

7. Meadows J, Kraut M, Guarnieri M, et al. Asymptomatic Chiari type I malformations identified on magnetic resonance imaging. J Neurosurg. 2000;92(6):920-926.

8. Strahle J, Muraszko KM, Kapurch J, et al. Natural history of Chiari malformation Type I following decision for conservative treatment. J Neurosurg Pediatr. 2011;8(2):214-221.

9. Arnautovic A, Splavski B, Boop FA, Arnautovic KI. Pediatric and adult Chiari malformation Type I surgical series 1965-2013: a review of demographics, operative treatment, and outcomes. J Neurosurg Pediatr. 2015;15(2): 161-177.

10. Robinson LC, Tubbs RS, Wellons JC III, Hankinson TC. Chiari malformations and syringohydromyelia. In: Ellenbogen RG, Sekhar LN, Kitchen N, eds. Principles of Neurological Surgery. 4th ed. Elsevier; 2018:170-182.

11. Benjamini Y, Drai D, Elmer G, et al. Controlling the false discovery rate in behavior genetics research. Behav Brain Res. 2001;125(1-2):279-284.

12. Ridgeway G, McCaffrey DF, Morral AR, et al. Toolkit for weighting and analysis of nonequivalent groups: a tutorial for the R TWANG package. RAND Corporation. Accessed July 30, 2020. https://www.rand.org/pubs/tools/TL136z1.html

13. Jiang E, Sha S, Yuan X, et al. Comparison of clinical and radiographic outcomes for posterior fossa decompression with and without duraplasty for treatment of pediatric Chiari I malformation: a prospective study. World Neurosurg. 2018; 110:e465-e472.

14. Limbrick DD. Posterior fossa decompression with and without duraplasty for Chiari type 1 malformation with syringomyelia. ClinicalTrials.gov. Accessed July 30, 2020. https:// clinicaltrials.gov/ct2/show/NCT02669836

15. Ellenbogen RG, Armonda RA, Shaw DW, Winn HR. Toward a rational treatment of Chiari I malformation and syringomyelia. Neurosurg Focus. 2000;8(3):E6.

16. Passias PG, Pyne A, Horn SR, et al. Developments in the treatment of Chiari type 1 malformations over the past decade. J Spine Surg. 2018;4(1):45-54.

17. Strahle J, Muraszko KM, Kapurch J, et al. Chiari malformation Type I and syrinx in children undergoing magnetic resonance imaging. J Neurosurg Pediatr. 2011;8(2): 205-213.

18. Kennedy BC, Nelp TB, Kelly KM, et al. Delayed resolution of syrinx after posterior fossa decompression without dural opening in children with Chiari malformation Type I. $J$ Neurosurg Pediatr. 2015;16(5):599-606. 
19. Hale AT, Adelson PD, Albert GW, et al. Factors associated with syrinx size in pediatric patients treated for Chiari malformation type I and syringomyelia: a study from the ParkReeves Syringomyelia Research Consortium. J Neurosurg Pediatr. 2020;25(6):629-639.

20. Ball MJ, Dayan AD. Pathogenesis of syringomyelia. Lancet. 1972;2(7781):799-801.

21. Gardner WJ, Angel J. The cause of syringomyelia and its surgical treatment. Cleve Clin Q. 1958;25(1):4-8.

22. Oldfield EH, Muraszko K, Shawker TH, Patronas NJ. Pathophysiology of syringomyelia associated with Chiari I malformation of the cerebellar tonsils. Implications for diagnosis and treatment. J Neurosurg. 1994;80(1):3-15.

23. Williams B. The distending force in the production of communicating syringomyelia. Lancet. 1970;2(7662):41-42.

24. Strahle J, Smith BW, Martinez M, et al. The association between Chiari malformation Type I, spinal syrinx, and scoliosis. J Neurosurg Pediatr. 2015;15(6):607-611.

25. Shen J, Tan H, Chen C, et al. Comparison of radiological features and clinical characteristics in scoliosis patients with Chiari I malformation and idiopathic syringomyelia: a matched study. Spine (Phila Pa 1976). 2019;44(23):1653-1660.

26. Kelly MP, Guillaume TJ, Lenke LG. Spinal deformity associated with Chiari malformation. Neurosurg Clin N Am. 2015; 26(4):579-585.

27. Mollano AV, Weinstein SL, Menezes AH. Significant scoliosis regression following syringomyelia decompression: case report. Iowa Orthop J. 2005;25:57-59.
28. Liu H, Yang C, Yang J, Xu Y. Pediatric Chiari malformation type I: long-term outcomes following small-bone-window posterior fossa decompression with autologous-fascia duraplasty. Exp Ther Med. 2017;14(6):5652-5658.

\section{Disclosures}

Dr. McGrath reports being a patent holder for and having ownership in EigenHealth, Inc.

\section{Author Contributions}

Conception and design: Wang, Ene, Ellenbogen. Acquisition of data: Wang, Ene, Collins, Bonow, McGrath. Analysis and interpretation of data: Wang, Ene, Barber, Ellenbogen. Drafting the article: Wang, Ene, Durfy. Critically revising the article: Wang, Ene, Bonow, Durfy, Barber, Ellenbogen. Reviewed submitted version of manuscript: all authors. Approved the final version of the manuscript on behalf of all authors: Wang. Statistical analysis: Wang, Ene, Barber. Administrative/technical/material support: Durfy. Study supervision: Wang, Ellenbogen.

\section{Correspondence}

Anthony C. Wang: University of California, Los Angeles, CA. acwang@mednet.ucla.edu. 